\title{
Wie ein rotierender Typ-IV-Pilus Archaeen das Schwimmen beibrachte
}

PATRICK TRIPP, SONJA-VERENA ALBERS

INSTITUT FÜR BIOLOGIE II, MIKROBIOLOGIE, UNIVERSITÄT FREIBURG

\section{Swimming motility in Archaea is mediated by the archaellum, a rotat- ing type IV pilus. Archaellum-based motility is the only known way of active locomotion in Archaea to date. Here, we describe the way of how the archaellum was discovered to be a unique structure in nature and highlight the latest findings on archaellar components.}

DOI: $10.1007 / \mathrm{s} 12268-020-1403-5$

(C) Die Autoren 2020

Viele Mikroorganismen nutzen ihre Fähigkeit zur gerichteten Bewegung, etwa um in nährstoffreichere Umgebungen zu gelangen und sich so einen Überlebensvorteil zu verschaffen. Dies trifft auch auf bewegliche Archaeen zu. Während in Bakterien mehrere verschiedene Arten von Fortbewegung beschrieben sind (schwimmen, gleiten, twitching - eine Art kriechender Bewegung), ist in Archaeen bisher nur das Schwimmen bekannt.

Die Struktur, die den Archaeen das Schwimmen ermöglicht, ist das Archaellum [1]. Archaellen sind filamentöse Zellanhänge mit einem Durchmesser von 10-14 Nanome-

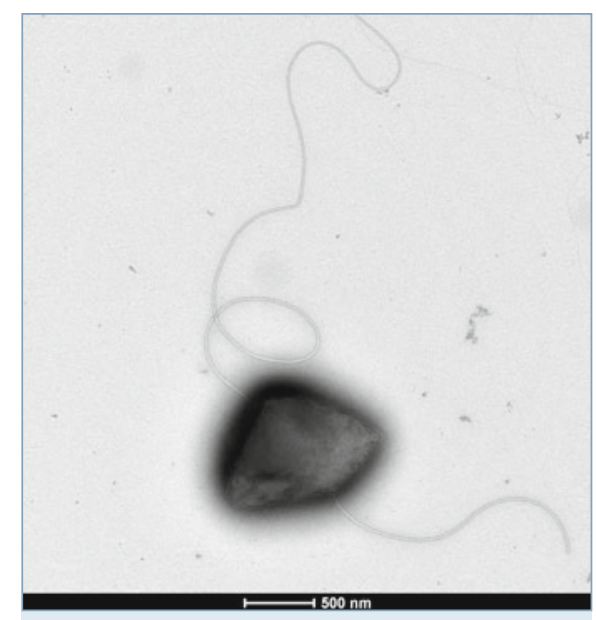

$\Delta$ Abb. 1: Elektronenmikroskopische Aufnahme von Sulfolobus acidocaldarius. Die Probe wurde mit Uranylacetat gefärbt. Zwei Archaellen sind deutlich sichtbar. tern und einer Länge von bis zu mehreren Mikrometern. Die Filamente bilden typischerweise eine rechtsgängige Helix, deren Rotation Vortrieb erzeugt. Die Anzahl von Archaellen pro Zelle schwankt stark: Während einige Spezies nur ein oder zwei Filamente ausbilden (z. B. Sulfolobus acidocaldarius, Abb. 1), besitzen andere Arten mehr als 50 Archaellen pro Zelle (z. B. Pyrococcus furiosus). Die Archaellen rotieren mit einer Rate von etwa 25 Hertz und erzeugen dabei M: Membran (verändert nach [9]). ein Drehmoment von $160 \mathrm{pN}$ nm [2]. Mithilfe des Archaellums bewegen sich Archaeen mit Geschwindigkeiten zwischen 3 und 590 Mikrometer pro Sekunde. Das entspricht bei den schnellsten Archaeen, Methanocaldococcus jannaschii und M. villosus, 400 bis 500 Körperlängen pro Sekunde (zum Vergleich: E. coli und Geparden erreichen nur das 20fache ihrer Körperlänge, [3]).

Obwohl das Archaellum wie das bakterielle Flagellum rotiert, um die Zelle anzutreiben, sind die Systeme nicht evolutionär verwandt. Das Archaellum ist ein Homolog des Typ-IV-Pilus und nutzt dementsprechend die ATP-Hydrolyse, um Energie für den Aufbau und die Rotation des Filaments bereitzustellen (Abb. 2, [4]).

Wie Typ-IV-Piline werden auch die Archaelline (z. B. ArlB), die Untereinheiten des Archaellum-Filaments, zunächst mit einem N-terminalen Klasse-III-Signalpeptid synthetisiert. Die Archaelline werden in die Membran inseriert, wo eine spezielle Peptidase (PibD oder ArlK) das Signalpeptid

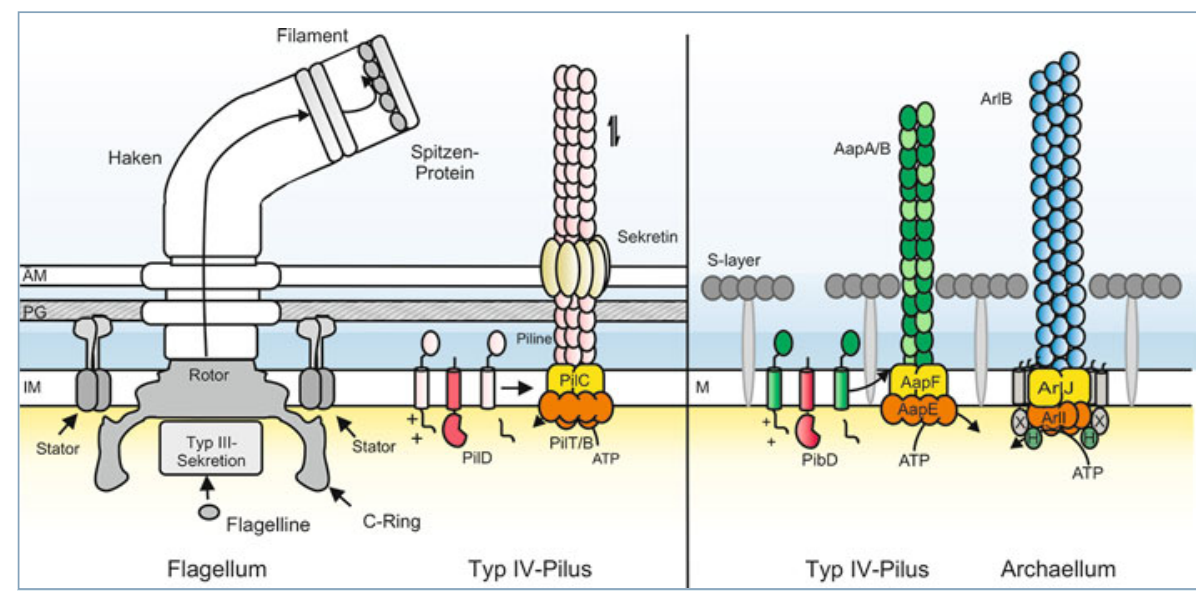

A Abb. 2: Vergleich von Flagellum und Typ-IV-Pilus von Bakterien (links) sowie des Typ-IV-Pilus von Archaeen und Archaellum (rechts). Das Flagellum wird durch Typ-III-Sekretionssysteme aufgebaut. Die Flagelline werden aus dem Cytoplasma über beide Membranen innerhalb des Filaments transportiert und dann an der Spitze des Filaments zusammengesetzt. Im Gegensatz dazu werden Typ-IV-Piline in der cytoplasmatischen Membran prozessiert und dann aus der Membran in das wachsende Filament integriert. Die zentrale Assemblierungsmaschine des Archaellums ähnelt den Komponenten aus Typ-IV-Pili (angedeutet mit denselben Farben). Der Unterschied ist, dass nach Assemblierung des Filaments die Motor-ATPase Arll das Filament rotiert und die Zellen so aktiv schwimmen können. AM: äußere Membran; PG: Peptidoglykan; IM: innere Membran; 


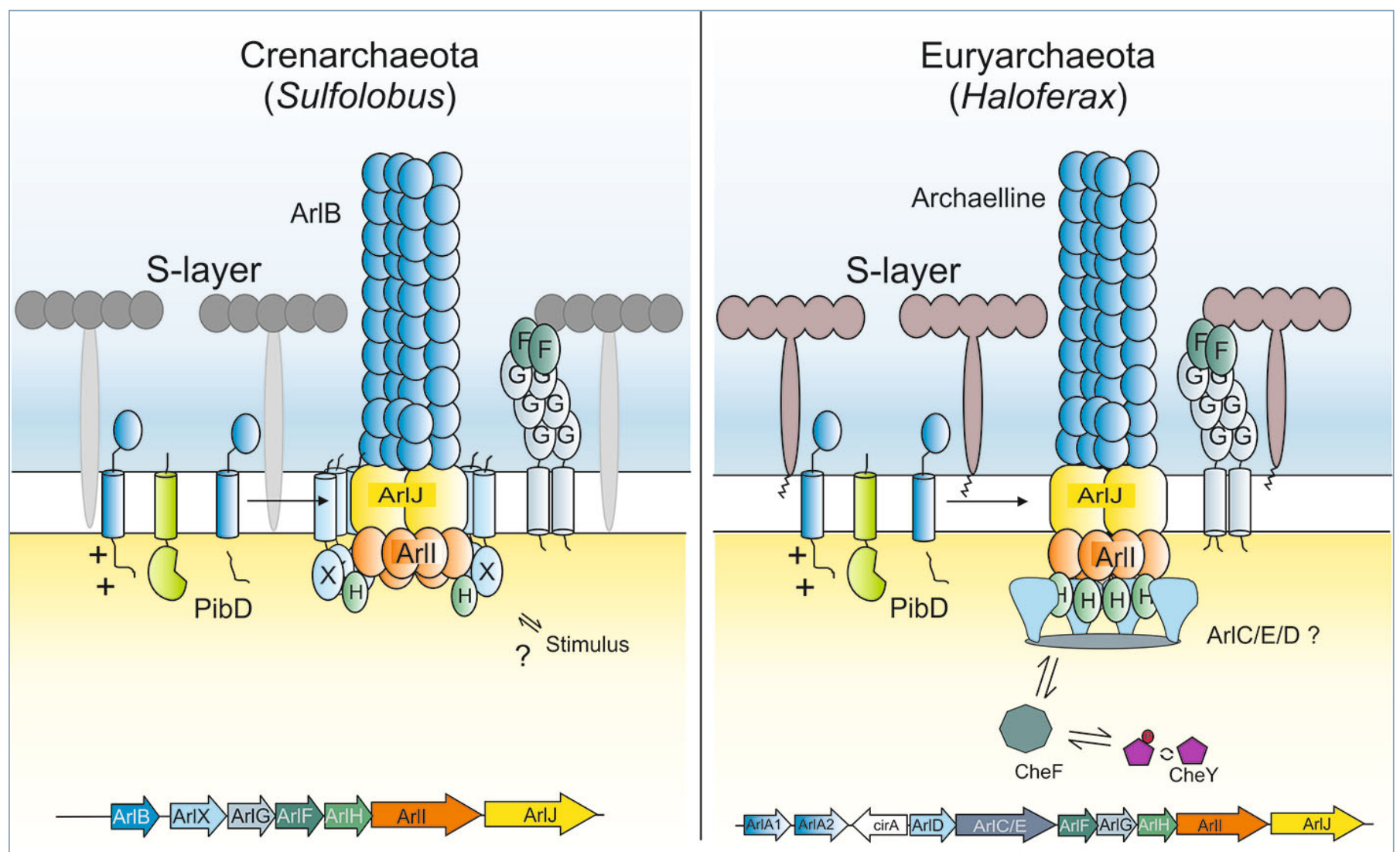

$\Delta$ Abb. 3: Vergleich des Archaellums bei Cren- und Euryarchaeota. In Crenarchaeota formt ArIX eine ringförmige Struktur, die den Motorkomplex umschließt. In Euryarchaeota findet man durchgängig die Proteine ArICDE, die die Kontaktstelle des Motors mit dem Chemotaxissystem darstellen. Ob das crenarchaeale Archaellum auf einen Stimulus hin seine Richtung verändert, ist unklar. CheF interagiert mit phosphoryliertem CheY und kann dann an den ArICED-Komplex binden, was zu einer Veränderung der Drehrichtung des Archaellum-Filaments führt. Als Beispiel für die typische Organisation des Archaellum-Operons sind unten die Operons von Sulfolubus acidocaldarius und Haloferax volcanii gezeigt.

erkennt und schneidet [5]. Sie entfernt lediglich das N-terminale Signalpeptid, während die hydrophobe Membrandomäne erhalten bleibt. Diese hydrophobe Domäne formt später den Kern des Filaments [6].

Das Membranprotein ArlJ formt die Basis für den Aufbau des Archaellums und ist homolog zu PilC, das die gleiche Aufgabe in Typ-IV-Pili übernimmt. (Die Nomenklatur der Archaellum-Gene wurde kürzlich von Fla auf Arl umgestellt [7].)

Die ATPase ArlI stellt sowohl die Energie für den Aufbau als auch für die spätere Rotation des Filaments zur Verfügung [8]. In Typ-IV-Pili finden sich die zwei ATPasen PilB und PilT, die jeweils für den Aufbau und das Einfahren des Pilus verantwortlich sind. Da das Archaellum aber nicht wieder eingezogen wird, benötigt das System nur das PilBHomolog ArlI.

Auf den ersten Blick scheint auch das lösliche Protein ArlH eine ATPase zu sein. Allerdings besitzt ArlH ein atypisches Walker-BMotiv, was dazu führt, dass Nukleotide zwar mit hoher Affinität gebunden, jedoch nicht oder nur extrem langsam hydrolysiert werden [9]. ArlH interagiert mit der ATPase ArlI, und diese Interaktion ist essenziell für den Aufbau des Archaellums. Strukturell zeigt ArlH hohe Ähnlichkeit zum Protein KaiC, das in Cyanobakterien für den circadianen Rhythmus verantwortlich ist. Dementsprechend wird vermutet, dass ArlH als Archaellum-Uhr fungiert, die es ermöglicht, dass ArII nach einer bestimmten Zeit vom „Aufbaumodus“ in den „Rotationsmodus“ wechselt.

In Crenarchaeoten (z. B. Sulfolobus acidocaldarius) bildet ArlX einen stabilisierenden Ring um den Motorkomplex des Archaellums und interagiert dabei direkt mit ArlH und ArlI. Euryarchaeoten (z. B. Haloferax volcanii) besitzen im arl-Operon $\operatorname{arlC}$, arlD und arlE anstelle von $\operatorname{arl} X$. Diese phylogenetische Varianz ist eine Anpassung, die es den Euryarchaeoten ermöglicht, ihr Archaellum mit dem bakteriellen Chemotaxissystem zu koppeln, das sie über horizontalen Gentransfer aufgenommen haben (Abb. 3, [10]).

Die Gene $\operatorname{arl} G$ und $\operatorname{arlF}$ finden sich in allen arl-Operons, aber in keinen anderen archae- ellen Typ-IV-Pili. Die Sekundärstruktur der Proteine erinnert stark an die von Archaellinen und Pilinen: eine lange, hydrophobe Helix am N-Terminus, gefolgt von mehreren $\beta$-Faltblättern in der C-terminalen Domäne. Im Gegensatz zu Archaellinen/Pilinen fehlt ArlG und ArlF allerdings das charakteristische Klasse-III-Signalpeptid. Dementsprechend war lange nicht klar, wie die Proteine zur Funktion des Archaellums beitragen. Wir konnten zeigen, dass die C-terminale lösliche Domäne von S. acidocaldarius ArlF an die Oberflächenschicht (S-layer, surface layer) bindet. In vielen Archaeen stellt diese Schicht den Hauptbestandteil der Zellwand dar, so auch in S. acidocaldarius. Diese Beobachtung brachte erste Erkenntnisse über die Rolle von ArlF im Archaellum mit sich. Wie auch im Flagellum benötigt das rotierende Archaellum einen Stator, der dafür sorgt, dass die vom Filament erzeugte Kraft auf die Zelle wirkt und zudem den rotierenden Komplex in der Membran stabilisiert [11]. Der Stator im Flagellum wird von MotA/B gebildet, wobei der Komplex von der 
Interaktion zwischen der Murein-Zellwand und MotB immobilisiert wird und somit als statischer Gegenpart zum Rotor wirken kann. Unsere Ergebnisse zeigen, dass ArlF analog dazu an die Oberflächenschicht (S-layer) bindet und so möglicherweise das Archaellum verankert.

Als Nächstes konnten wir nachweisen, dass ArlG und ArlF einen Komplex bilden, dessen Ausbildung für den Aufbau des Archaellums benötigt wird. Dieser Komplex sitzt wahrscheinlich an der Spitze eines Filaments, das von ArlG ausgebildet wird. Um dieses Filament zu bilden und den ArlG/ F-Komplex zur Oberflächenschicht zu bringen, muss ArlG seine Membrandomäne abtrennen. Wie dieser Reifeprozess abläuft und welche Proteine daran beteiligt sind, ist bis jetzt unklar. Interessanterweise konnten wir aber zeigen, dass der Verlust der Membrandomäne unabhängig von der Signalpeptidase abläuft, die ArlB prozessiert [11].

Es wird wichtig sein, noch weitere Details über die Interaktion der verschiedenen Archaellum-Proteine zu lernen, um zu verstehen, wie das Archaellum-Filament assembliert wird und dann rotieren kann.

\section{Literatur}

[1] Albers SV, Jarrell KF (2018) The Archaellum: an update on the unique archaeal motility structure. Trends Microbiol 26:351-362

[2] Iwata S, Kinosita Y, Uchida N et al. (2019) Motor torque measurement of Halobacterium salinarum archaellar suggests a general model for ATP-driven rotary motors. Commun Biol 2:199

[3] Herzog B, Wirth R (2012) Swimming behavior of selected species of Archaea. Appl Environ Microbiol 78:1670-1674
[4] Shahapure R, Driessen RPC, Haurat MF et al. (2014) The archaellum: a rotating type IV pilus. Mol Microbiol 91:716-723 [5] Albers SV, Szabó Z, Driessen AJM (2003) Archaeal homolog of bacterial type IV prepilin signal peptidases with broad substrate specificity. J Bacteriol 185:3918-3925 [6] Poweleit N, Ge P, Nguyen HH et al. (2016) CryoEM structure of the Methanospirillum hungatei archaellum reveals structural features distinct from the bacterial flagellum and type IV pili. Nat Microbiol 2:16222

[7] Pohlschröder M, Pfeiffer F, Schulze S et al. (2018) Archaeal cell surface biogenesis. FEMS Microbiol Rev 42: 694-717

[8] Reindl S, Ghosh A, Williams GJ et al. (2013) Insights into FlaI functions in archaeal motor assembly and motility from structures, conformations, and genetics. Mol Cell 49:10691082

[9] Chaudhury P, Neiner T, D'Imprima E et al. (2016) The nucleotide-dependent interaction of FlaH and FlaI is essentia for assembly and function of the archaellum motor. Mol Microbiol 99:674-685

[10] Wuichet K, Zhulin IB (2010) Origins and diversification of a complex signal transduction system in prokaryotes. Sci Signal 3:ra50

[11] Tsai CL, Tripp P, Sivabalasarma S et al. (2020) The structure of the periplasmic FlaG-FlaF complex and its essential role for archaellar swimming motility. Nat Microbiol 5: 216-225
Funding: Open Access funding provided by Projekt DEAL.

Open Access: Dieser Artikel wird unter der Creative Commons Namensnennun 4.0 International Lizenz veröffentlicht, welche die Nutzung, Vervielfältigung, erlaubt, sofern Sie den/die ursprünglichen Autor(en) und die Quelle ordnungsgemäß nennen, einen Link zur Creative Commons Lizenz beifügen und angeben, ob Änderungen vorgenommen wurden. Die in diesem Artikel enthaltenen Bilder und sonstiges Drittmaterial unterliegen ebenfalls der genannten Creative Commons Lizenz, sofern sich aus der Abbildungslegende nichts anderes ergibt. Sofern das betreffende Material nicht unter der genannten Creative Commons Lizenz steht und die betreffende Handlung nich nach gesetzlichen Vorschriften erlaubt ist, ist fur die oben aufgeführten Weiterverwendungen des Materials die Einwilligung des jeweiligen

\section{Korrespondenzadresse:}

Prof. Dr. Sonja-Verena Albers

Institut für Biologie II - Mikrobiologie

Molekularbiologie der Archaeen

Universität Freiburg

Schänzlestraße 1

D-79104 Freiburg

sonja.albers@biologie.uni-freiburg.de

www.archaellum.org,

www.ag-albers.uni-freiburg.de

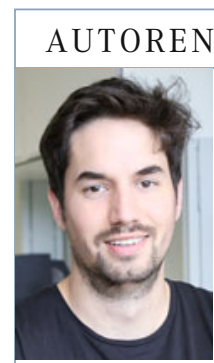

Patrick Tripp

2010-2014 Bachelorstudium Biologie und Masterstudium Molekular- und Zellbiologie an der Universität Marburg. 2015-2019 Doktorand an der Universität Freiburg in der Forschungsgruppe von Prof. Dr. S.-V. Albers.

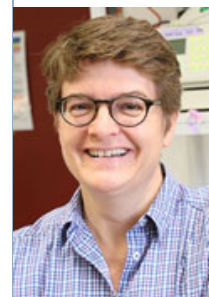

Sonja-Verena Albers

1991-1996 Biologiestudium an der Universität Würzburg. 1997-2001 Doktorarbeit an der Universität in Groningen, Niederlande; dort 2001-2002 Postdoc. 2003-2005 VENI-Stipendium und 2006-2008 VIDI-Gruppenleiterin, Universität Groningen. 2008-2014 Arbeitsgruppenleiterin zur Molekularbiologie der Archaeen am MaxPlanck-Institut für terrestrische Mikrobiologie in Marburg. Seit 2014 W3-Professorin für Mikrobiologie an der Universität Freiburg. 